\title{
RNA binding protein HuR promotes osteosarcoma cell progression via suppressing the miR-142-3p/HMGA1 axis
}

\author{
WEICHENG PAN, JINHUI PANG, BIN JI, ZHEN WANG, CHENGWEI LIU, YAN CHENG and LEI ZHANG
}

Department of Orthopedics, Shanghai Putuo District Central Hospital, Shanghai 200062, P.R. China

Received October 9, 2017; Accepted April 6, 2018

DOI: $10.3892 / 01.2018 .8855$

\begin{abstract}
The present study aimed to study the roles and underlying mechanisms of human antigen $\mathrm{R}(\mathrm{HuR})$ in osteosarcoma (OS) cell progression. It was determined that the HuR mRNA and protein levels were significantly upregulated in OS tissues, compared with that in normal adjacent tissues. HuR expression was negatively associated with miR-142-3p expression, but positively with High Mobility Group AT-Hook 1 (HMGA1). Additionally, knockdown of HuR inhibited OS cells viability, epithelial-mesenchymal transition and promoted cell apoptosis. HuR was determined to harbor binding sites on HMGA1, directly binding to HMGA1, increasing HMGA1 mRNA stability and expression. Notably, the promotion of HuR on HMGA1 expression was attenuated via miR-142-3p overexpression, and miR-142-3p could directly bind to HMGA1 3'untranslated region (UTR). Furthermore, HMGA1 3'UTR with a mutated miR-142-3p binding site did not respond to $\mathrm{HuR}$ alterations. Finally, the inhibition of HuR knockdown was attenuated or even reversed via HMGA1 overexpression; therefore, the results of the present study indicated that RNA binding protein HuR may facilitate OS cell progression via competitively binding to HMGA1 with miR-142-3p.
\end{abstract}

\section{Introduction}

Osteosarcoma (OS) is a common bone malignant tumor with a high degree of malignancy (1). Of patients with OS, $\sim 75 \%$ are between 15 and 25 years old, and the primary treatment options include surgical section and chemoradiotherapy (2). However, the prognosis of patients with OS is poor, and the long-term survival rate of patients with metastasis or recurrence is $<20 \%$ (3). Therefore, investigating the molecular mechanisms underlying OS progression may contribute to

Correspondence to: Dr Zhen Wang, Department of Orthopedics, Shanghai Putuo District Central Hospital, 409 Meiling North Road, Shanghai 200062, P.R. China

E-mail: yungang_wu@sina.com

Key words: HuR, HMGA1, miR-142-3p, osteosarcoma, RNA binding protein the development of novel prognostic biomarkers and targeted therapies.

A recent study has indicated that epigenetic modulation serves important roles in various physiological and pathological processes (4). RNA binding proteins (RBPs) and microRNAs (miRNAs) are essential epigenetic modulators $(5,6)$. RBPs specifically bind to and enhance mRNA stability, thus increasing mRNA expression (5). miRNAs, a type of non-coding and single-strand RNA containing 18-22 nucleotides, regulate mRNAs at the post-transcriptional level via binding to complementary sequences in untranslated regions (UTRs) of target mRNAs, inducing their degradation or repressing translation (6). Human antigen R (HuR), as a $\mathrm{RBP}$, has been demonstrated to facilitate the progression of various tumor types (7), and binds to mRNA 3'UTR, thus repressing the inhibition of miRNAs on mRNA expression (8). However, the roles and associated underlying mechanisms of $\mathrm{HuR}$ in OS progression are unclear.

miR-142-3p could function as a potential tumor suppressor in OS via targeting High mobility Group AT-Hook 1 (HMGA1) (9). Long non-coding RNA MALAT1 promotes OS progression by regulating HMGB1 expression via miR-142-3p and miR-129 (10). In the present study, bioinformatics suggested that HuR binds to HMGA1. Notably, HMGA1 may promote thyroid cancer and colon cancer proliferation and invasion $(11,12)$. Thus, it was hypothesized that HuR promotes OS progression via binding to and enhancing HMGA1 mRNA expression, and whether HuR could promote HMGA1 expression via miR-142-3p was further explored.

The results of the present study demonstrated that HuR directly binds to HMGA1, and enhances HMGA1 mRNA stability and expression. The promotion of HuR in OS progression and HMGA1 expression may be attenuated via miR-142 overexpression. Notably, HMGA1 3'UTR with a mutated miR-142-3p binding site did not respond to HuR overexpression. Collectively, these results elucidated the roles and associated underlying mechanisms of HuR in OS progression.

\section{Materials and methods}

Clinical tissues, cells culture and bioinformatics analysis. The human OS cell line MG63 was purchased from the American Type Culture Collection (Manassas, VA, USA). MG63 cells were cultured in Dulbecco's modified Eagle's medium (Gibco; Thermo Fisher Scientific, Inc., Waltham, 
MA, USA) containing 10\% fetal bovine serum (FBS, Gibco; Thermo Fisher Scientific, Inc.), penicillin and streptomycin at $37^{\circ} \mathrm{C}$ under a humidified atmosphere containing $5 \% \mathrm{CO}_{2}$. A total of 32 OS tumor samples, paired with adjacent normal tissues, were obtained from 12 females and 20 males patients aged 19-61 who underwent surgery at Shanghai Putuo District Central Hospital (Shanghai, China) between February 2014 and January 2017. Approval from the Institute Research Ethics Committee of Shanghai Putuo District Central Hospital was obtained for the use of these clinical materials for research purposes and written informed consent was obtained from patients prior to surgery. Bioinformatics analysis (http://starbase.sysu.edu.cn/index.php) was used to predict the potential targets of RNA binding protein HuR.

Reverse transcription-quantitative polymerase chain reaction $(R T-q P C R)$. mRNA expression was confirmed via RT-qPCR analysis. Briefly, total RNA was extracted from the tissues and the MG63 cell line using TRIzol ${ }^{\circledR}$ reagent (Invitrogen; Thermo Fisher Scientific, Inc.). The first strand cDNA was synthesized using the RevertAid ${ }^{\mathrm{TM}}$ First Strand cDNA Synthesis kit (Thermo Fisher Scientific, Inc.) according to the manufacturer's protocols. The mRNA expression levels were determined on the ABI Prism 7500 Detection system (Applied Biosystems; Thermo Fisher Scientific, Inc.) with Universal SYBR ${ }^{\circledR}$ Green mix (Vazyme, Piscataway, NJ, USA). The denaturing process was $95^{\circ} \mathrm{C}$ for $5 \mathrm{~min}$, the annealing process was $58^{\circ} \mathrm{C}$ for $30 \mathrm{sec}$ and the elongation process was $72^{\circ} \mathrm{C}$ for $30 \mathrm{sec}$. There were 35 cycles of this RT-qPCR performed. Relative mRNA expression was calculated with the $2^{-\Delta \Delta C q}$ method (13). The primers for RT-qPCR were indicated in Table I. Melting curve analysis and 1\% agarose gel electrophoresis were used to check amplification specificity and length of PCR products. GAPDH and U6 snRNA served as an internal control for mRNA and miRNA expression, respectively.

Western blot analysis. Following different treatments, proteins were extracted using the protein extraction kit (Nanjing KeyGen Biotech Co., Ltd., Nanjing, China). Protein concentration was examined via the Bradford assay. The detailed procedure was referred to in the previous study (9). A total of $30 \mu \mathrm{g}$ of protein was analyzed on $10 \%$ SDS-PAGE and electrotransferred to PVDF membranes (Millipore, Bedford, Massachusetts). The membranes were blocked in 5\% non-fat dried milk for $60 \mathrm{~min}$ at room temperature. Primary antibodies against HuR (cat. no. ab136542), HMGA1 (cat. no. ab129153), Cleaved-caspase3 (cat. no. ab49822), caspase3 (cat. no. ab13847), epithelial (E)-cadherin (cat. no. ab1416), Vimentin (cat. no. ab8978) and $\beta$-actin (cat. no. ab8227) were purchased from Abcam (Cambridge, UK), and the dilution ratio of all antibodies was 1:5,000. Following incubating with primary antibodies, blots were washed with tris-buffered saline with $0.5 \%$ Tween 20 and incubated with a peroxidase-conjugated secondary Goat Anti-Rabbit (cat. no., A0208, Beyotime Institute of Biotechnology; dilution rate: 1:5,000) and Goat Anti-Mouse antibody (cat. no., A0216, Beyotime Institute of Biotechnology; dilution rate: 1:5,000), and chemiluminescence was detected using an enhanced chemiluminescence kit (Thermo Fisher Scientific, Inc.) followed by exposure in Bio-Rad ChemiDoc ${ }^{\mathrm{TM}}$
MP system (Bio-Rad Laboratories, Inc., Hercules, CA, USA). The protein expression level was normalized to $\beta$-actin.

Cell viability analysis. Cell Counting Kit-8 (CCK-8; Shanghai Yeasen Biotechnology Co., Ltd., Shanghai, China) was used to analyze the cell viability rate of cells with different treatments, in which cells without HuR knockdown were used as control. In brief, cells were suspended and 3,000 cells/well were seeded into 96-well plates, following culturing with DMEM medium with 10\% FBS (Gibco; Thermo Fisher Scientific, Inc.) for 24, 48 and 72 h. CCK-8 was added into the DMEM, following the manufacturer's protocol. The absorbance was detected at $450 \mathrm{~nm}$. The cell viability rate was presented with the value relative to the control group. Experiments were repeated at least three times.

Cell apoptosis assay. Cell apoptotic rate was analyzed using the Annexin V-FITC and propidium iodide (PI) kit (Beyotime Institute of Biotechnology, Haimen, China) via flow cytometry. Cells with different treatments were stained with Annexin V-FITC and PI, followed with flow cytometry analysis using a BD FACSCanto II (BD Biosciences, Franklin Lakes, NJ, USA), following the manufacturer's protocol. FlowJo software (version 10.0.7; FlowJo LLC, Ashland, OR, USA) was used to analyze the data which were expressed as cell percentage.

Luciferase reporter assay. HMGA1 3'UTR sequences were inserted into the PMIR-Reporter plasmid (cat. no., AM5795; Thermo Fisher Scientific, Inc.), denoted as Luc-HMGA1-wt. PCR primers for Luc-HMGA1-wt were indicated in Table I. PMIR-Reporter plasmid holding HMGA1 3'UTR sequences with the mutated binding site of miR-142-3p was obtained with the Site-directed Gene Mutagenesis kit (Beyotime Institute of Biotechnology) and noted as Luc-HMGA1-mut. These aforementioned plasmids were co-transfected with $\beta$-gal control plasmid plus Lenti-HuR infection into MG63 cells using Lipofectamine ${ }^{\circledR} 2000$ reagent (Invitrogen; Thermo Fisher Scientific, Inc.), according to the manufacturer's protocol. A total of $48 \mathrm{~h}$ later, cells were lysed with Reporter lysis buffer (cat. no, E397A; Promega Corporation, Madison, WI, USA) and luciferase activity was measured with VivoGlo Luciferin kit (cat. no., P1041; Promega Corporation) using a luminometer (Thermo Fisher Scientific, Inc.). The luciferase activity was normalized to $\beta$-gal activity.

Lentivirus package. shRNAs against HuR and a scramble non-targeting shRNA were purchased from Santa Cruz Biotechnology, Inc. (Dallas, TX, USA). The shRNA sequences were inserted into pLKO.1 (Sigma-Aldrich, Merck KGaA), termed as Lenti-HuR-shRNA. Additionally, HMGA1 and HuR sequences were inserted into pLVX-IRES-ZsGreen1, termed as Lenti-HMGA1 and Lenti-HuR, respectively. PCR primers for plasmids construction were mentioned in Table I. Lentivirus were packaged in HEK293T cells via co-transfecting Lenti-HuR-shRNA or Lenti-HMGA1 or Lenti-HuR with pCMV-dR8.2 and pMD2.G constructs using Lipofectamine 2000. Following $72 \mathrm{~h}$, the supernatants were collected and ultrafiltrated. The virus supernatants $(10 \mu \mathrm{l}$ in $10^{8} \mathrm{TU} / \mathrm{ml}$ ) were added to MG63 cells with $2 \mu \mathrm{g} / \mathrm{ml}$ Polybrene (Genomeditech, Shanghai, China). 
Table I. PCR primer sequences.

Gene Sequence (5'-3')

HuR RT-qPCR forward

HuR RT-qPCR reverse

HMGA1 RT-qPCR forward

HMGA1 RT-qPCR reverse

Ki67 RT-qPCR forward

Ki67 RT-qPCR reverse

E-cadherin RT-qPCR forward

E-cadherin RT-qPCR reverse

Vimentin RT-qPCR forward

Vimentin RT-qPCR reverse

GAPDH RT-qPCR forward

GAPDH RT-qPCR reverse

Luc-HMGA1-wt forward

Luc-HMGA1-wt reverse

Lenti-HuR forward

Lenti-HuR reverse

Lenti-HMGA1 forward

Lenti-HMGA1 reverse

ATTGTATGTGGTCTCCGCTGTTTG
TTTCTTTTGGGTTGAGCCTTTTT
CCCGCCCACCCACGCATACACACA
GCCCCCAAACCAAAAGCCCAGAGA
GTGCTCAACAACTTCATTTCCAAC
AACACATTTCCTCCAAAACTCTCT
ATGGCTTCCCTCTTTCATCTCCTG
TTCATAGTTCCGCTCTGTCTTTGG
TCAGAATATGAAGGAGGAAATGGC
TCAGGGAGGAAAAGTTTGGAAGAG
CGGAGTCAACGGATTTGGTCGTAT
AGCCTTCTCCATGGTGGTGAAGAC
AAGAAAAACCTTCCCGGTGCAATCG
CAAGTAACTGCAAATAGGAAACCAG
ATGTCTAATGGTTATGAAGACCACA
TTATTTGTGGGACTTGTTGGTTTTG
ATGAGTGAGTCGAGCTCGAAGTCCA
TCACTGCTCCTCCTCCGAGGACTCC

HuR, human antigen R; HMGA1, High Mobility Group AT-Hook 1; Lenti-HuR, HuR sequences inserted into pLVX-IRES-ZsGreen1; Lenti-HMGA1, HMGA1 sequences inserted into pLVX-IRES-ZsGreen1; E-cadherin, epithelial-Cadherin; Luc-HMGA1-wt, wild type HMGA1 3'UTR sequences inserted into the PMIR-Reporter plasmid; RT-qPCR, reverse transcription-quantitative polymerase chain reaction.

mRNA stability assay. HuR was knocked down via infecting with Lenti-HuR-shRNA for $48 \mathrm{~h}$ at $37^{\circ} \mathrm{C}$. Then, $5 \mu \mathrm{g} / \mathrm{ml}$ ActD (ApexBio, Houston, TX, USA) was added to inhibit the de novo RNA synthesis. Total RNA was collected at 2, 4 and $6 \mathrm{~h}$ and mRNA expression was determined via RT-qPCR analysis under the same conditions as previously described. The mRNA half-life was determined by comparing to the mRNA level prior to adding ActD.

RNA immunoprecipitation (RIP) assay. MG63 cells with or without HuR knockdown were lysed with $25 \mathrm{mM}$ Tris- $\mathrm{HCl}$ buffer (pH 7.5) and 100 U/ml RNase inhibitor (Sigma-Aldrich; Merck KGaA, Darmstadt, Germany), and then incubated with protein-A Sepharose beads (Genescript, Nanjing, China) precoated with $2 \mu \mathrm{g}$ anti-HuR antibody (as previously stated, dilution rate, 1:100), or control rabbit IgG (cat no. ab191867, Abcam, dilution rate, 1:100) was used for negative control for $3 \mathrm{~h}$ at $4^{\circ} \mathrm{C}$. The RNA-protein complexes were pulled-down by protein A/G agarose beads (Genescript) and RNA was extracted with TRIzol, followed by detecting the HMGA1 expression level with RT-qPCR assay, as previously described. This experiments were repeated at least three times.

Statistical analysis. All data were obtained from at least three independent experiments $(n \geq 3)$, and presented as the mean \pm standard deviation. Datasets with only two groups were analyzed using Student's t-test. Differences among multiple groups were analyzed using one-way analysis of variance with the Tukey's post-hoc test, and $\mathrm{P}<0.05$ was considered to indicate a statistically significant difference.

\section{Results}

HuR expression level is upregulated in OS tissues compared with normal adjacent tissues. Firstly, the expression level of HuR in OS tissues and normal adjacent tissues was detected. RT-qPCR and western blot analysis demonstrated that HuR expression was increased in OS tissues, compared with normal adjacent tissues (Fig. 1A and B). An identical result was obtained in a further immunohistochemical assay (Fig. 1C). These results indicated that HuR promoted OS progression.

Knockdown of HuR enhances OS cell apoptosis, and inhibits cell viability and the epithelial-mesenchymal transition (EMT) process. Since the expression level of HuR was upregulated in OS tissues, HuR expression was knocked down in OS cells. RT-qPCR and western blot analysis confirmed the knockdown efficiency of Lenti-HuR-shRNA (Fig. 2A and B). CCK-8 analysis indicated that HuR knockdown significantly inhibited MG63 cell viability (Fig. 2C) and the expression of proliferation marker Ki67 (Fig. 2D). A further cell apoptosis assay indicated that the knockdown of HuR facilitated cell apoptosis (Q2 and Q3) in MG63 cells (Fig. 2E) and promoted the expression of the apoptosis executor, cleaved caspase 3 (Fig. 2F). Additionally, HuR knockdown notably suppressed the EMT process in MG63 cells, characterized by the downregulation of the expression of the mesenchymal marker Vimentin and upregulation of the expression of the epithelial marker E-cadherin (Fig. 2G and H). These results demonstrated that HuR knockdown inhibited OS progression. 


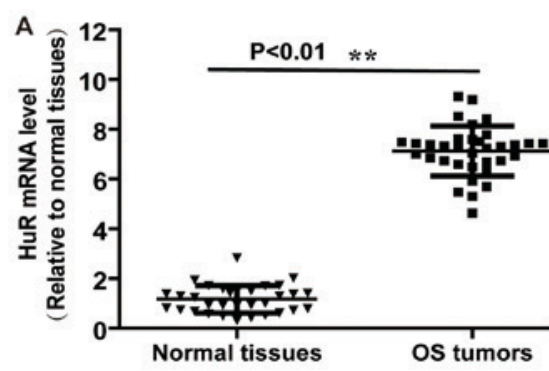

B
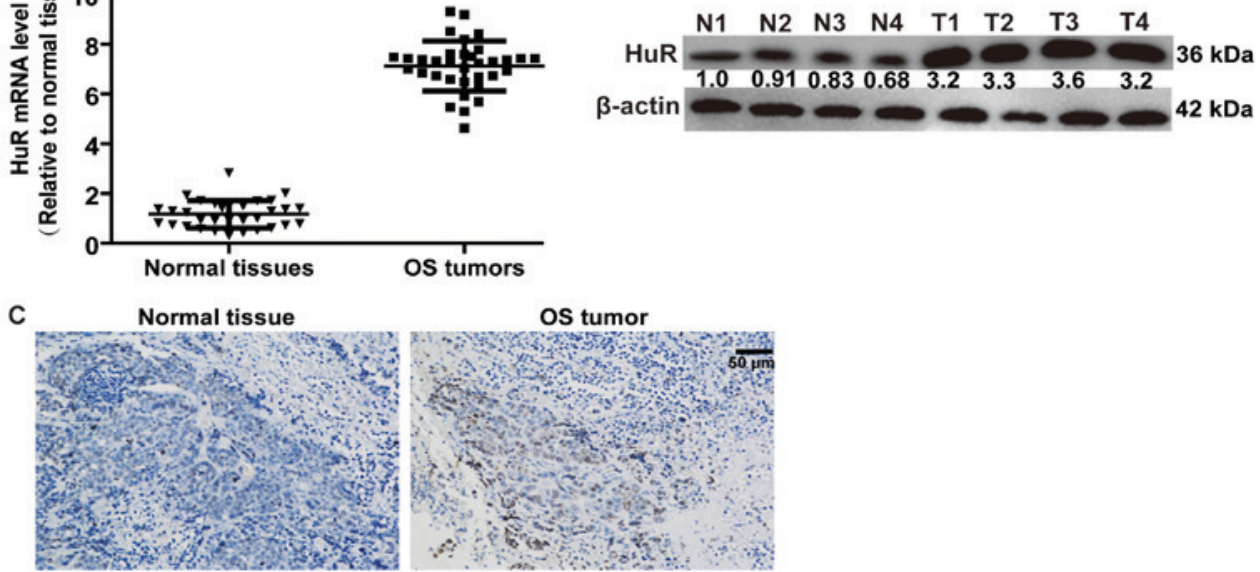

Figure 1. HuR expression level is upregulated in OS tissues, compared with that in normal adjacent tissues. (A) mRNA level of HuR was examined in OS and normal adjacent tissues via RT-qPCR ( $\mathrm{n}=32$ ) analysis. (B) The protein expression level of HuR was detected in OS and normal adjacent tissues via western blot analysis. (C) An immunohistochemistry assay was performed to detect the HuR expression level in OS and normal adjacent tissues, and represented figures were presented. ${ }^{* *} \mathrm{P}<0.01$ vs. normal tissues. OS, osteosarcoma; HuR, human antigen R.
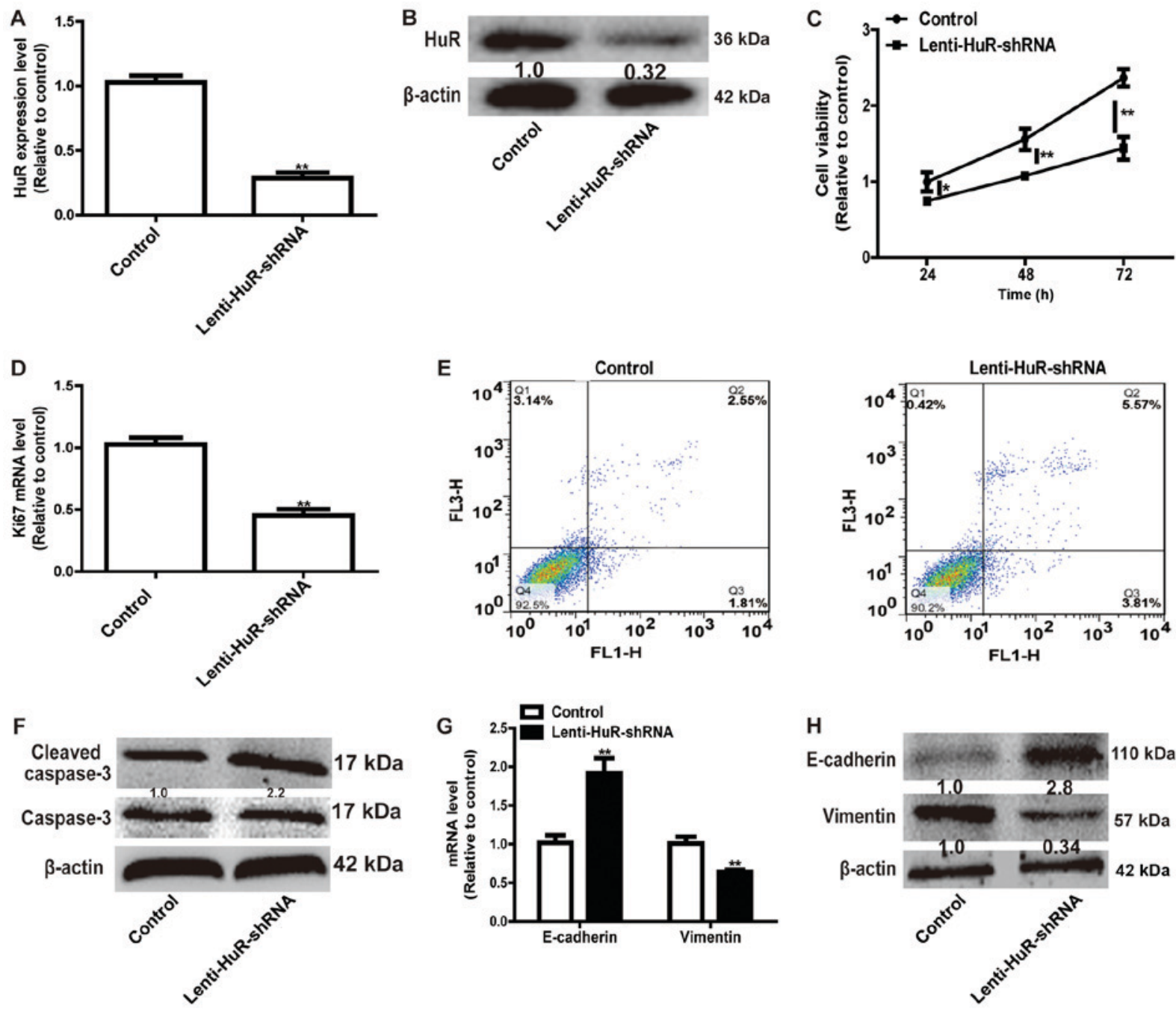

Figure 2. Knockdown of HuR enhances OS cell apoptosis, and inhibits cell viability and the EMT process. Knockdown efficiency of Lenti-HuR-shRNA was validated via (A) RT-qPCR and (B) western blot analysis. (C) Cells with or without HuR knockdown were subjected to an MTT assay to examine cell viability. (D) The mRNA expression level of proliferation marker ki67 was measured in the cells depicted in Fig. 2C. (E) Cell apoptosis assay was performed to detect the apoptotic rate of the cells depicted in Fig. 2C. (F) The protein expression level of apoptotic executor Cleaved caspase-3 was tested in cells depicted in Fig. 2C. EMT markers (E-cadherin and Vimentin) (G) mRNA and (H) protein expression levels were measured in cells depicted in Fig. $2 \mathrm{C}$. ${ }^{*} \mathrm{P}<0.05{ }^{, * *} \mathrm{P}<0.01$ vs. control. Lenti-HuR-shRNA, shRNA sequences inserted into pLKO.1; E-Cadherin, epithelial-Cadherin; HuR, human antigen R. 

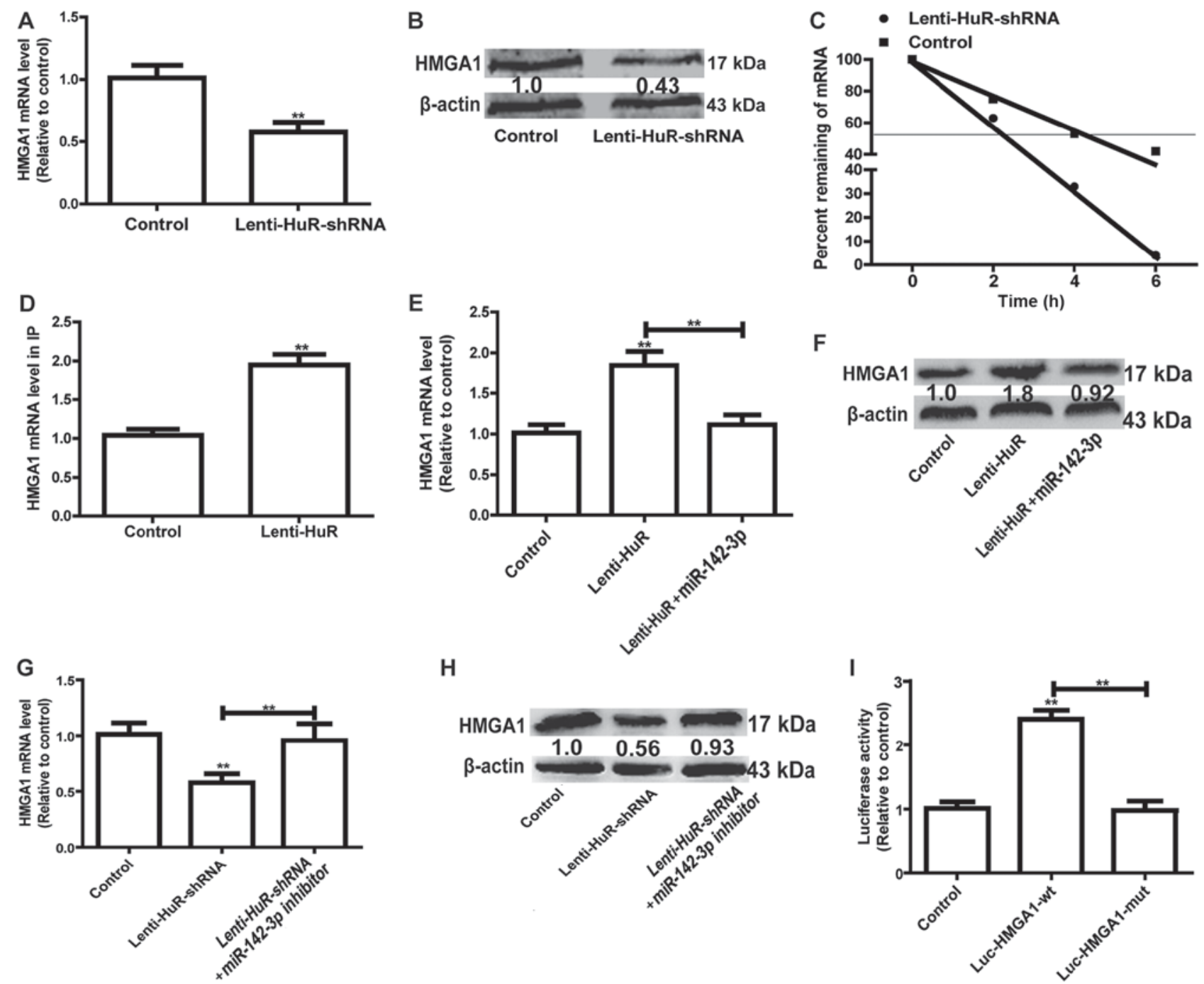

Figure 3. HuR represses the inhibition of miR-142-3p on HMGA1 expression. (A) The mRNA expression level of HMGA1 was detected in cells with or without Lin28A. (B) The protein expression level of HMGA1 was examined in the cells depicted in Fig. 3A. (C) The mRNA expression level of HMGA1 was measured at the indicated times with the addition of ActD $(5 \mu \mathrm{g} / \mathrm{ml})$ in the cells depicted in Fig. 3A. (D) RT-qPCR was used to measure HMGA1 abundance presented in the HuR-IP materials following the RIP assay in cells with or without HuR overexpression. (E) mRNA and (F) protein expression levels of HMGA1 were detected in cells with HuR overexpression, and with or without miR-142-3p overexpression. (G) mRNA and (H) protein expression levels of HMGA1 were detected in cells with HuR knockdown, and with or without miR-142-3p knockdown. (I) A luciferase reporter assay was performed to detect the effect of HuR overexpression on luciferase activities of the control vector, Luc-HMGA1-wt and Luc-HMGA1-mut. ${ }^{* *} \mathrm{P}<0.01$ vs. control unless indicated otherwise. Lenti-HuR-shRNA, shRNA sequences inserted into pLKO.1; HuR, human antigen R; HMGA1, High Mobility Group AT-Hook 1; RIP, RNA immunoprecipitation; Lenti-HuR, HuR sequences inserted into pLVX-IRES-ZsGreen1; Luc-HMGA1-wt, wild type HMGA1 3'UTR sequences inserted into the PMIR-Reporter plasmid; Luc-HMGA1-mut, Luc-HMGA1-wt with mutated miR-142-3p binding site.

$H u R$ represses the inhibition of miR-142-3p on HMGA1 expression. As HuR belongs to the group of RBPs, which bind to mRNA, and enhance mRNA stability and expression (7), targets were identified via bioinformatics analysis (http://starbase.sysu.edu.cn/index.php). HMGA1 has been identified to promote the progression of OS (11). RT-qPCR and western blot analysis demonstrated that the knockdown of HuR significantly decreased HMGA1 expression in MG63 cells (Fig. 3A and B). Furthermore, HMGA1 mRNA stability was reduced with HuR knockdown (Fig. 3C). Thus, it was speculated that HuR may directly bind to HMGA1 in MG63 cells. HuR expression was induced in MG63 cells, with the HuR-binding complex knocked down via the HuR antibody, followed by examination of the bound mRNAs via RT-qPCR. RIP results indicated that HuR had a significantly greater association with HMGA1 compared with the control (Fig. 3D). Since the miR-142-3p/HMGA1 axis has been confirmed in OS cells (9), whether HuR was involved in the miR-142-3p/HMGA1 regulatory pathway in MG63 cells was further investigated. As depicted in Fig. 3E and $F$, overexpression of miR-142-3p significantly attenuated the promoter effect of HuR on HMGA1 expression. By contrast, knockdown of miR-142-3p reversed the inhibitory effects of HuR knockdown on HMGA1 expression (Fig. 3G and $\mathrm{H}$ ). Additionally, mutation of the miR-142-3p binding site on HMGA1 3'UTR partially reversed the enhancement of HuR on the luciferase activity of Luc-HMGA1-wt (Fig. 3I). Overall, these results indicated that HuR promoted HMGA1 expression via competitively binding to HMGA1 3'UTR with miR-142-3p. 

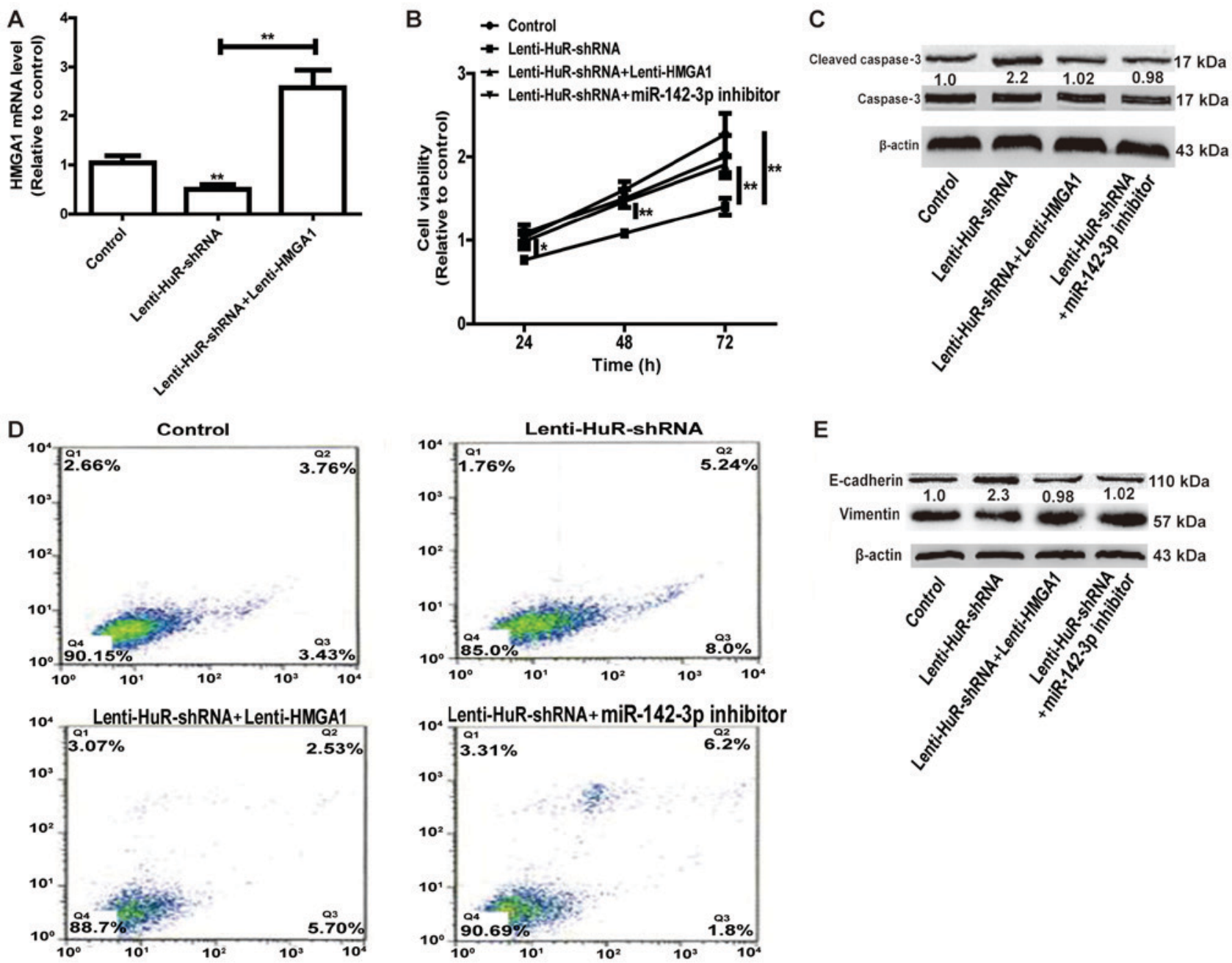

E
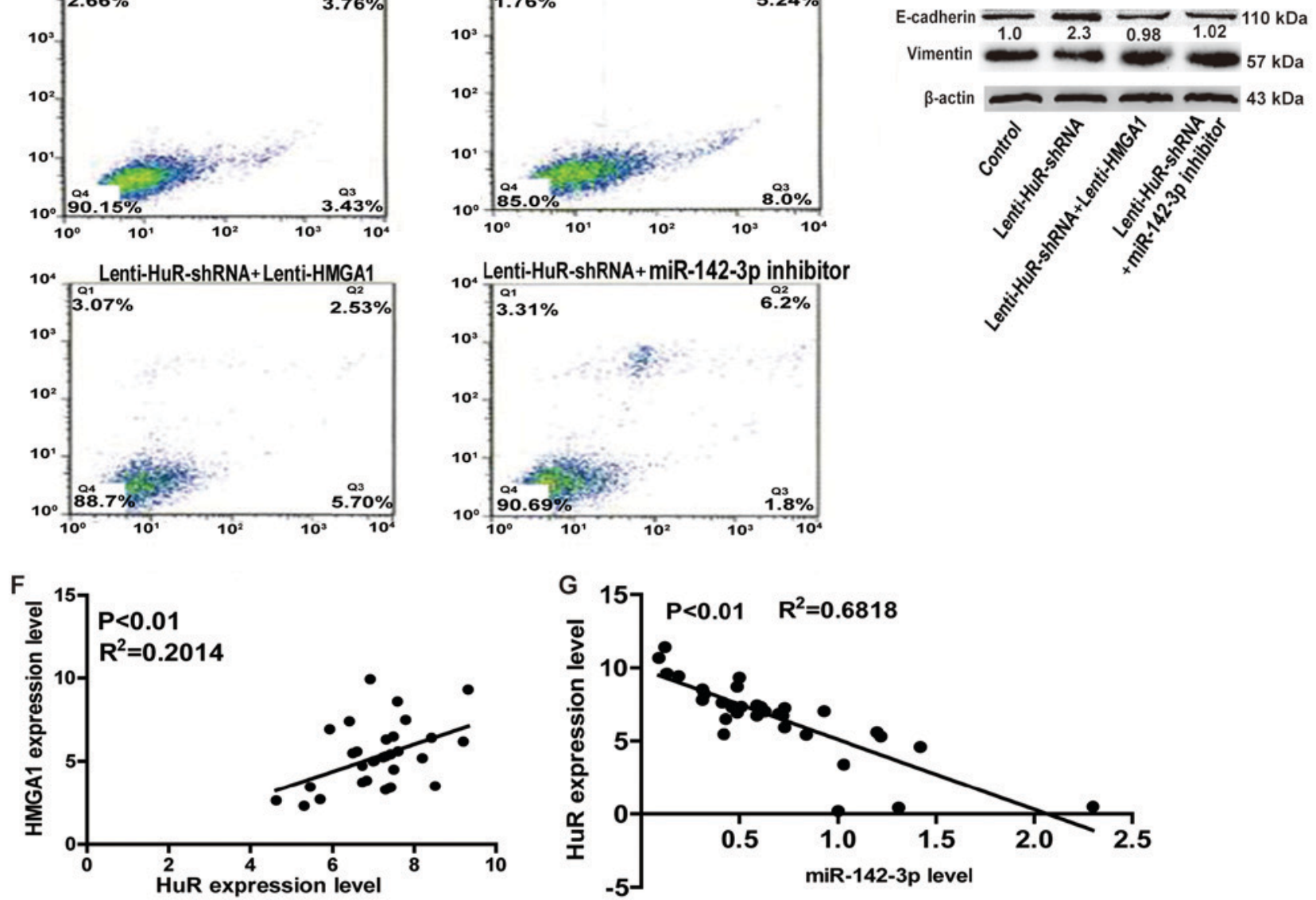

Figure 4. HuR promotes OS progression in a miR-142-3p/HMGA1 axis-dependent manner. (A) The HMGA1 expression level was detected in HuR knockdown cells with or without HMGA1 overexpression. (B) Cell viability was examined in HuR knockdown cells with HMGA1, or with or without miR-142-3p overexpression. (C) The protein expression level of apoptotic executor Cleaved caspase-3 was measured in the cells depicted in Fig. 4B. (D) Cell apoptosis was detected in the cells depicted in Fig. 4B. (E) The EMT markers (E-cadherin and Vimentin) protein levels were measured in the cells depicted in Fig. 4B. The Lin28A expression level exhibited positive or negative association with (F) HMGA1 or (G) miR-142-3p expression level, respectively. "P<0.05, ${ }^{* *} \mathrm{P}<0.01$ vs. control unless indicated otherwise. Lenti-HuR-shRNA, shRNA sequences inserted into pLKO.1; HuR, human antigen R; HMGA1, High Mobility Group AT-Hook 1; Lenti-HuR, HuR sequences inserted into pLVX-IRES-ZsGreen1; Lenti-HMGA1, HMGA1 sequences inserted into pLVX-IRES-ZsGreen1.

HuR promotes OS progression in a miR-142-3p/HMGAl axis-dependent manner. Whether miR-142-3p/HMGA1 axis was involved in the promotion of HuR in OS progression was further investigated. As depicted in Fig. 4A, infection with Lenti-HMGA1 significantly upregulated the HuR expression level, reversing the suppressive effects of HuR knockdown on HMGA1 expression. Cell viability and apoptosis assays indicated that overexpression of HMGA1 or transfection with miR-142-3p inhibitor attenuated the inhibition of HuR knockdown on cell proliferation and promotion on cell apoptosis (Fig. 4B-D). Additionally, HMGA1 overexpression or miR-142-3p knockdown notably attenuated or even reversed EMT process inhibited by HuR knockdown (Fig. 4E). Notably, HuR expression was positively associated with HMGA1 expression, and negatively associated with miR-142-3p expression in OS tissues (Fig. 4F and G). Therefore, the results of the present study results indicated that HuR promoted OS progression at least partly through the miR-142-3p/HMGA1 axis. 


\section{Discussion}

HuR is overexpressed in a number of cancer types, including malignant brain tumors (8) and pancreatic cancer cells (14). However, its role in OS remains unclear. In the present study, it was elucidated that HuR contributed to cell viability and inhibited cell apoptosis in MG63 cells.

Although HuR have been frequently investigated, the majority of its functions are via its targets (15). A previous study indicated that RBPs bind to mRNA 3'UTR competitively with miRNAs, including transformer $2 \beta$ and miR-204, regulating apoptosis through competitive binding to 3'UTR of B-cell lymphoma-2 mRNA (16). Additionally, DND microRNA-mediated repression inhibitor 1 promotes breast cancer apoptosis via stabilizing Bim mRNA in a miR-221 binding site (17). These results demonstrate that $\mathrm{HuR}$ may facilitate OS progression via competitively binding to mRNA with miRNAs. A bioinformatics assay demonstrated that HMGA1 was a potential target of HuR. Previous studies have indicated the promotion of HMGA1 in OS progression (9), and that HMGA1 modulates autophagy in cancer cells (18). These results confirmed the oncogenic roles of HMGA1. Notably, the results of the present study indicated that HMGA1 overexpression attenuated the inhibition of HuR knockdown on OS progression, indicating that $\mathrm{HuR}$ exerts its effects at least partly through HMGA1. Furthermore, as the miR-142-3p/HMGA1 axis has been associated with OS progression (9), and it was determined that mutation of the binding site of miR-142-3p on HMGA1 sequences prevented the promotion of HuR via HMGA1, these results indicated that miR-142-3p is involved in the interaction between $\mathrm{HuR}$ and HMGA1, and HuR may competitively bind to HMGA1 3'UTR via miR-142-3p.

To the best of our knowledge, this is the first study demonstrating the roles and associated mechanisms underlying HuR in OS progression. As HMGA1 may be targeted by other RBPs, including insulin growth factor 2 (19), there may be other RBPs, which are involved in the regulation of HMGA1 in OS progression, which require further investigation. However, since the oncogenic roles of HuR have been identified in other tumor types, it was hypothesized that analyzing other functions, and more detailed mechanisms underlying HuR/HMGA1 axis during OS development may provide significant insights into gene regulatory networks and their clinical implications for OS.

\section{Acknowledgements}

Not applicable.

\section{Funding}

No funding was received.

\section{Availability of data and materials}

All data generated or analyzed during the current study are included in this published article.

\section{Authors' contributions}

WP and ZW designed the study. WP, JP and LZ analyzed the data. WP, JP, BJ, YC and CL performed the experiments. WP and $\mathrm{LZ}$ wrote the manuscript.

\section{Ethics approval and consent to participate}

Approval from the Institute Research Ethics Committee of Shanghai Putuo District Central Hospital was obtained for the use of these clinical materials for research purposes and written informed consent was obtained from patients prior to the study start.

\section{Consent for publication}

Not applicable.

\section{Competing interests}

The authors declare no competing interests.

\section{References}

1. Picci P: Osteosarcoma (osteogenic sarcoma). Orphanet J Rare Dis 2: 6, 2007.

2. Heare T, Hensley MA and Dell'Orfano S: Bone tumors: Osteosarcoma and Ewing's sarcoma. Curr Opin Pediatr 21: 365-372, 2009.

3. Weeden S, Grimer RJ, Cannon SR, Taminiau AH and Uscinska BM; European Osteosarcoma Intergroup: The effect of local recurrence on survival in resected osteosarcoma. Eur J Cancer 37: 39-46, 2001

4. Hu J, Li X, Guo X, Guo Q, Xiang C, Zhang Z, Xing Y, Xi T and Zheng L: CCR2 3'UTR functions as a competing endogenous RNA to inhibit breast cancer metastasis. J Cell Sci 130: 3399-3413, 2017.

5. Nahas GR, Murthy RG, Patel SA, Ganta T, Greco SJ and Rameshwar P: The RNA-binding protein Musashi 1 stabilizes the oncotachykinin $1 \mathrm{mRNA}$ in breast cancer cells to promote cell growth. FASEB J 30: 149-159, 2016.

6. Fang L, Du WW, Yang X, Chen K, Ghanekar A, Levy G, Yang W, Yee AJ, Lu WY, Xuan JW, et al: Versican 3'-untranslated region (3'-UTR) functions as a ceRNA in inducing the development of hepatocellular carcinoma by regulating miRNA activity. FASEB J 27: 907-919, 2013.

7. Zhang W, Vreeland AC and Noy N: RNA-binding protein HuR regulates nuclear import of protein. J Cell Sci 129: 4025-4033, 2016.

8. Steinmeyer N, Doller A, Biyanee A, Brauss T, Schmid T, Pfeilschifter J and Eberhardt W: Lymphotoxin $\alpha$, a novel target of posttranscriptional gene regulation by HuR in HepG2 cells. FEBS Lett 589: 1943-1950, 2015.

9. Xu G, Wang J, Jia Y, Shen F, Han W and Kang Y: MiR-142-3p functions as a potential tumor suppressor in human osteosarcoma by targeting HMGA1. Cell Physiol Biochem 33: 1329-1339, 2014.

10. Liu K, Huang J, Ni J, Song D, Ding M, Wang J, Huang X and Li W: MALAT1 promotes osteosarcoma development by regulation of HMGB1 via miR-142-3p and miR-129-5p. Cell Cycle 16: 578-587, 2017.

11. Zhong J, Liu C, Zhang QH, Chen L, Shen YY, Chen YJ, Zeng X, $\mathrm{Zu} X \mathrm{X}$ and Cao RX: TGF- $\beta 1$ induces HMGA1 expression: The role of HMGA1 in thyroid cancer proliferation and invasion. Int J Oncol 50: 1567-1578, 2017.

12. Yang Q, Wang X, Tang C, Chen X and He J: H19 promotes the migration and invasion of colon cancer by sponging miR-138 to upregulate the expression of HMGA1. Int J Oncol 50: 1567-1578, 2017.

13. Livak KJ and Scmittgen TD: Analysis of relative gene expression data using real-time quantitative PCR and the 2(-Delta Delta $\mathrm{C}(\mathrm{T})$ ) method. Methods 25: 402-408, 2001. 
14. Romeo C, Weber MC, Zarei M, DeCicco D, Chand SN, Lobo AD, Winter JM, Sawicki JA, Sachs JN, Meisner-Kober N, et al: HuR contributes to TRAIL resistance by restricting death receptor 4 expression in pancreatic cancer cells. Mol Cancer Res 14: 599-611, 2016.

15. Muralidharan R, Panneerselvam J, Chen A, Zhao YD, Munshi A and Ramesh R: HuR-targeted nanotherapy in combination with AMD3100 suppresses CXCR4 expression, cell growth, migration and invasion in lung cancer. Cancer Gene Ther 22: 581-590, 2015.

16. Kuwano Y, Nishida K, Kajita K, Satake Y, Akaike Y, Fujita K, Kano S, Masuda K and Rokutan K: Transformer $2 \beta$ and miR-204 regulate apoptosis through competitive binding to 3' UTR of BCL2 mRNA. Cell Death Differ 22: 815-825, 2015

17. Cheng F, Pan Y, Lu YM, Zhu L and Chen S: RNA-binding protein Dnd1 promotes breast cancer apoptosis by stabilizing the Bim mRNA in a miR-221 binding site. Biomed Res Int 2017: 9596152, 2017.
18. Conte A, Paladino S, Bianco G, Fasano D, Gerlini R, Tornincasa M, Renna M, Fusco A, Tramontano D and Pierantoni GM: High mobility group A1 protein modulates autophagy in cancer cells. Cell Death Differ 24: 1948-1962, 2017.

19. Dai N, Ji F, Wright J, Minichiello L, Sadreyev R and Avruch J: IGF2 mRNA binding protein-2 is a tumor promoter that drives cancer proliferation through its client mRNAs IGF2 and HMGA1. Elife 6: e27155, 2017.

This work is licensed under a Creative Commons Attribution-NonCommercial-NoDerivatives 4.0 International (CC BY-NC-ND 4.0) License. 